\title{
Autonomous Manipulation of Deformable Objects based on Teleoperated Demonstrations
}

\author{
Matthias Rambow, Thomas Schauß, Martin Buss, and Sandra Hirche
}

\begin{abstract}
While humans can manipulate deformable objects smoothly and naturally, this is still a challenge for autonomous robots due to the complex object dynamics. The presence of rigid environment constraints and altering contact phases between the deformable object, the manipulator, and the environment makes this problem even more challenging. This paper presents a framework for deformable object manipulation that makes use of a single human demonstration of the task. The recorded trajectories are automatically segmented into a sequence of haptic control primitives involving contact with the rigid environment and vision-guided grasp primitives. The recorded motion/force trajectories serve as reference for a compliant control scheme in contact situations. In order to cope with positioning uncertainties a variable admittance control is proposed. The proposed approach is validated in an experimental mounting task for a deformable linear object with multiple re-grasping. The task is demonstrated with a multimodal teleoperation system and transfered to a robotic platform with a pair of seven degrees of freedom manipulators.
\end{abstract}

\section{INTRODUCTION}

While the manipulation of rigid objects is a well studied field in autonomous robotics, handling of deformable objects is still challenging. The control laws for rigid object manipulation cannot directly be applied to flexible object manipulation tasks, mainly due to the changing shape of the object. Additionally, manipulation planning for deformable objects requires complex calculations as the object dynamics are typically approximated by FEM models which have a large number of states. In this work we address the problem of inserting a deformable linear object (DLO). DLOs are a special class of flexible objects with the property that their length is a multitude of their diameter. The solution of this problem would be very beneficial for industrial production processes, where the manipulation of DLOs like cables, wires, or tubes is still mostly performed by human workers.

The existing work on manipulation of DLOs can be separated into approaches that i) apply modeling and planning methods and ii) approaches, which utilize human demonstrations. Prior work mainly focuses on modeling of DLOs and planning algorithms for their manipulation. In [1] a physical static model for DLOs based on differential geometry is presented The model is extended to a dynamic model for the two-dimensional case in [2]. But the complexity of the model prevents using it for re-planning during manipulation. In [3] a motion planner for the bi-manual manipulation of ropes is

The authors are with the Institute of Automatic Control Engineering (LSR) at the Technische Universität München, Germany. \{rambow, schauss, mb, hirche\} at tum.de

This work is supported in part within the DFG excellence initiative research cluster Cognition for Technical Systems - CoTeSys (www.cotesys.org) proposed. In an experimental setup this planner is used to tie several knots. However, this approach is limited to knotting problems. In general, one can conclude that, especially in the domain of deformable objects, mathematical models describing the objects are computationally expensive. This makes an on-line re-planning during the execution infeasible. Furthermore, the existing planners are often restricted to special domains.

This suggests to investigate programming by demonstration methods. They make direct use of an expert's experience and transfer it to a technical system. In [4] a skill controller in form of an event-driven hybrid controller is realized in order to solve a hose insertion task. The control parameters are identified from human demonstrations. Changes in the contact state are used as events for the switching policy, but the events are manually labeled in the continuous data profiles. An approach for an automatic program generation for a "DLO-in-hole" task is presented in [5]. There, a sequence of topological contact states of the DLO and the environment is extracted from a user demonstration in a virtual environment. One restriction here is that a very precise virtual model of the DLO is needed. Furthermore, positioning inaccuracies are difficult to handle. In summary, programming by demonstration techniques offer a promising way of teaching manipulation skills. However, they are in general not robust against position uncertainties in the environment and initial state differences between demonstration and autonomous execution.

The contribution of this paper is a framework for autonomous deformable object manipulation based on the programming by demonstration paradigm. Instead of using a pure replay of the complicated manipulation task demonstrated by a human on a trajectory level, we suggest to find a sequence of controllers for which the demonstrated trajectory is an attractor. An automatic segmentation approach decomposes task into several haptic primitives and basic skills, including vision-guided grasping. In order to cope with position uncertainties a variable admittance control scheme is proposed for the haptic control primitives. The approach is experimentally evaluated with a bi-manual DLO insertion task on a robot with two anthropomorphic 7-DoF arms.

The remainder of this paper is structured as follows: Section II briefly defines the manipulation problem considered in this work. In Section III we propose our framework for the autonomous execution of the manipulation task and describe the methods used. Section IV introduces the experimental setup followed by the experimental evaluation. 


\section{Problem Definition}

In this paper we consider bi-manual manipulation tasks which involve multiple contact phases of the robot with the deformable object as well as with the rigid environment through the deformable object. The task is demonstrated once by a human expert. In order to provide the human demonstrator with a realistic impression of the task, a teleoperation system with visuo-haptic feedback is used for the demonstration. Hereby, we assume the demonstrating user does not grasp or manipulate simultaneously with both arms or perform any manipulation steps useless for accomplishing the task. The tele-operated robot is also used for the autonomous task execution. This has the advantage that constraints of the robotic system and avoidance of unwanted collisions with the environment are implicitly considered when executing the task autonomously.

The output of the demonstration is the time series $\mathbf{P}=\left\{\mathbf{p}_{t}=\left(\mathbf{x}_{t}, \mathbf{f}_{t}\right): t \in[0, T]\right\}$ for each of the two arms representing the trajectory of the end-effector's pose $\mathbf{x} \in \mathbb{R}^{3} \times \mathbb{R} \mathbb{P}^{3}$ and wrench $\mathbf{f} \in \mathbb{R}^{6}$ in the robot coordinate frame from the initial time 0 to $T$. In order to be able to grasp the DLO at the same position aiming at a reproducible behavior during manipulation, we store the time series $Y=\left\{y_{t}: t \in[0, T]\right\}$ of grasping points $y \in \mathbb{R}$ expressed in the DLO coordinate system. The DLO coordinate is defined as the distance from the starting point to the specified point along the DLO curve. If the tube is not grasped at time $t$, this is indicated by $y_{t}=\emptyset$.

Due to the presence of uncertainties a direct position replay of trajectories recorded from a complex demonstration is likely to fail. As the task imposes multiple contact phases of the robot with the DLO, the time series $\mathbf{P}$ is decomposed into chunks $\mathbf{P}_{i}=\left\{\mathbf{p}_{t}: t \in\left[t_{i, \text { start }}, t_{i, \text { end }}\right]\right\}$ from contact state to contact state. This yields a sequence of control primitives necessary to accomplish the task, i.e. the task plan. A control primitive is defined as a reference trajectory (or setpoint) and an associated control which makes the reference an attractor. As a consequence of the decomposition of the task into a sequence of control primitives we face two major research challenges to be addressed in this paper: i) How to design the control for the haptic control primitives (i.e. involving contact), which renders the demonstrated trajectory a stable attractor despite positioning uncertainties, and ii) how to automatically extract the task plan in terms of a sequence of control primitives from the demonstrated trajectories.

\section{Autonomous DLO-Manipulation Framework}

For bi-manual DLO manipulation tasks we identify four control primitives for each of the two robot manipulators: the grasp primitive, the support primitive for re-grasping, the manipulate primitive and the idle primitive. The grasp, support and idle primitives are quite common and can be solved by standard techniques like position control, either visual guided for grasping or based on a reference pose. However, the parametrization of the haptic control primitive for manipulation is not trivial and is described in the following.

\section{A. Haptic Control Primitives with Variable Admittance}

In the idealized case of exactly the same conditions as in the demonstration, a simple position control scheme would suffice to track the desired pose given by the demonstrated trajectory. However, due to uncertainties in the platform and consequently manipulator positioning with respect to the task this approach is infeasible. Therefore, we propose a compliant control scheme which renders the demonstrated pose/wrench trajectory an attractor. The haptic control primitive consists then of the desired (demonstrated) trajectory $\mathbf{P}_{i}$ and the compliant feedback control scheme, proposed in this section.

The task of inserting a DLO results in unilateral constraints in some directions in the workspace. The constrained directions are not known beforehand but directly result from the uncertainties during task execution. Compliant control schemes with high stiffness provide high position accuracy in free space, however, high forces may occur in contact in the direction of the position error. In those directions a lower stiffness is desirable. Therefore, here we propose an errordependent position/force-based admittance control scheme to track position and force reference trajectories while at the same time providing compliance to cope with uncertainties. Hereby we assume control errors are caused from contact of the DLO with the rigid environment. The control renders the virtual admittance

$$
\mathbf{M}_{\mathbf{x}}(\mathbf{e}(t)) \ddot{\mathbf{x}}(t)+\mathbf{D}_{\mathbf{x}}(\mathbf{e}(t)) \dot{\mathbf{x}}(t)+\mathbf{K}_{\mathbf{x}}(\mathbf{e}(t)) \mathbf{e}_{\mathbf{x}}(t)=\mathbf{e}_{\mathbf{f}}(t),
$$

where $\mathbf{x} \in \mathbb{R}^{3} \times \mathbb{R P}^{3}$ is the $6 D$-pose of the end-effector, $\mathbf{e}_{\mathbf{x}}=\left(\begin{array}{ll}\mathbf{e}^{T} & \mathbf{e}_{r}^{T}\end{array}\right)^{T}$ the $6 D$ position error composed of the translational error $\mathbf{e}(t)=\mathbf{x}_{p}(t)-\mathbf{x}_{p, d}(\tau(t)) \in \mathbb{R}^{3}$ and the rotational error $\mathbf{e}_{r}(t) \in \mathbb{R P}^{3}$. The error in the wrench is denoted by $\mathbf{e}_{\mathbf{f}}(t)=\mathbf{f}(t)-\mathbf{f}_{d}(\tau(t)) \in \mathbb{R}^{6}$, where $\mathbf{f}(t) \in \mathbb{R}^{6}$ is the wrench measured with a force-torque sensor located at the end-effector. The error-dependent time warping $\tau(t)$ is explained later. The desired pose/wrench point $\left(\mathbf{x}_{d}, \mathbf{f}_{d}\right) \in \mathbf{P}_{i}$ is part of a position/force trajectory recorded during the demonstration, and

$$
\begin{aligned}
\mathbf{M}_{\mathbf{x}}(\mathbf{e}(t)) & =\operatorname{diag}\left(\mathbf{M}(\mathbf{e}(t)), \mathbf{M}_{o}\right) \\
\mathbf{D}_{\mathbf{x}}(\mathbf{e}(t)) & =\operatorname{diag}\left(\mathbf{D}(\mathbf{e}(t)), \mathbf{D}_{o}\right) \\
\mathbf{K}_{\mathbf{x}}(\mathbf{e}(t)) & =\operatorname{diag}\left(\mathbf{K}(\mathbf{e}(t)), \mathbf{K}_{o}\right)
\end{aligned}
$$

are the rendered inertia, damping and stiffness, respectively. Hereby, $\mathbf{M}(\mathbf{e}(t)), \mathbf{D}(\mathbf{e}(t))$ and $\mathbf{K}(\mathbf{e}(t)) \in \mathbb{R}^{3 \times 3}$ are the translational components and $\mathbf{M}_{o}, \mathbf{D}_{o}$ and $\mathbf{K}_{o} \in \mathbb{R}^{3 \times 3}$ the rotational components. Note that for simplicity of notation we introduce the error-dependent adaptation of the admittance only in the translational degrees of freedom. An extension to the rotational degrees of freedom is feasible. The parameters for the rendered admittance (1) are adapted depending on the translational control error according to

$$
\begin{aligned}
\mathbf{M}(\mathbf{e}(t)) & =\overline{\mathbf{M}}+\mathbf{R}(\mathbf{e}(t))\left(\mathbf{A}_{m}\|\mathbf{e}(t)\|\right) \mathbf{R}^{-1}(\mathbf{e}(t)) \\
\mathbf{D}(\mathbf{e}(t)) & =\overline{\mathbf{D}}+\mathbf{R}(\mathbf{e}(t))\left(\mathbf{A}_{d}\|\mathbf{e}(t)\|\right) \mathbf{R}^{-1}(\mathbf{e}(t)) \\
\mathbf{K}(\mathbf{e}(t)) & =\overline{\mathbf{K}}+\mathbf{R}(\mathbf{e}(t))\left(\mathbf{A}_{k}\|\mathbf{e}(t)\|\right) \mathbf{R}^{-1}(\mathbf{e}(t))
\end{aligned}
$$




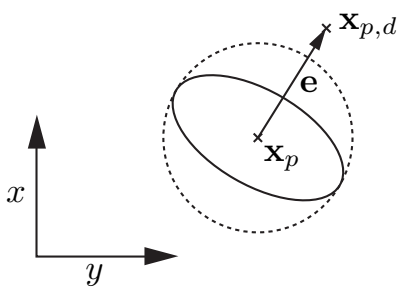

Fig. 1. Effect of the adaptation law for the rendered stiffness (solid ellipsoid) compared to the basic stiffness (dashed ellipsoid).

under the constraints $\mathbf{M}(\mathbf{e}(t)) \succ 0, \mathbf{D}(\mathbf{e}(t)) \succ 0$ and $\mathbf{K}(\mathbf{e}(t)) \succ 0$. Here, $\mathbf{R}(\mathbf{e}(t))$ is a rotation matrix that aligns the world coordinate frame's $\mathrm{x}$-axis with $\mathbf{e}(t)$, i.e

$$
\begin{aligned}
R(\mathbf{e}(t)) & =\left(\mathbf{r}_{x}(\mathbf{e}(t)) \quad \mathbf{r}_{y}(\mathbf{e}(t)) \quad \mathbf{r}_{z}(\mathbf{e}(t))\right) \text { with } \\
\mathbf{r}_{x}(\mathbf{e}(t)) & =\mathbf{e}(t) /\|\mathbf{e}(t)\| \\
\mathbf{r}_{y}(\mathbf{e}(t)) & =\left(\begin{array}{lll}
1 & 0 & 0
\end{array}\right)^{T} \times \mathbf{r}_{x}(\mathbf{e}(t)) \text { and } \\
\mathbf{r}_{z}(\mathbf{e}(t)) & =\mathbf{r}_{x}(\mathbf{e}(t)) \times \mathbf{r}_{y}(\mathbf{e}(t))^{1} .
\end{aligned}
$$

Further, $\overline{\mathbf{M}}, \overline{\mathbf{D}}$ and $\overline{\mathbf{K}}$ are the basic mass, damping, and stiffness matrices. Additionally we define the matrices

$$
\begin{aligned}
\mathbf{A}_{m} & =\left(\begin{array}{ccc}
p_{m} & 0 & 0 \\
0 & 0 & 0 \\
0 & 0 & 0
\end{array}\right), \mathbf{A}_{d}=\left(\begin{array}{ccc}
-p_{d} & 0 & 0 \\
0 & 0 & 0 \\
0 & 0 & 0
\end{array}\right), \\
\mathbf{A}_{k} & =\left(\begin{array}{ccc}
-p_{k} & 0 & 0 \\
0 & 0 & 0 \\
0 & 0 & 0
\end{array}\right),
\end{aligned}
$$

where $p_{m}, p_{d}$ and $p_{p} \geq 0$ are appropriate adaptation gains. For the sake of simplicity we will consider uncoupled, isotropic admittance properties, i.e. $\overline{\mathbf{M}}=\operatorname{diag}\{m\}$, $\overline{\mathbf{D}}=\operatorname{diag}\{d\}$ and $\overline{\mathbf{K}}=\operatorname{diag}\{k\}$ with fixed parameters $m, d$ and $k$. Note that in this case one can write, e.g. for the stiffness matrix

$$
\begin{aligned}
\mathbf{K}(\mathbf{e}(t)) & =\overline{\mathbf{K}}+\mathbf{R}(\mathbf{e}(t))\left(\mathbf{A}_{k}\|\mathbf{e}(t)\|\right) \mathbf{R}^{-1}(\mathbf{e}(t)) \\
& =\mathbf{R}(\mathbf{e}(t))\left(\overline{\mathbf{K}}+\mathbf{A}_{k}\|\mathbf{e}(t)\|\right) \mathbf{R}^{-1}(\mathbf{e}(t))
\end{aligned}
$$

and positive definiteness can be achieved by saturating the matrix entries according to

$$
\left(\overline{\mathbf{K}}+\mathbf{A}_{k}\|\mathbf{e}(t)\|\right)=\left(\begin{array}{ccc}
\left.\max \left\{k_{\min }, k-p_{k}\|\mathbf{e}(t)\|\right)\right\} & 0 & 0 \\
0 & k & 0 \\
0 & 0 & k
\end{array}\right)
$$

with the minimum stiffness $k_{\min }$. The same property holds for $\mathbf{M}(t), \mathbf{D}(t)$. Fig. 1 exemplary shows the effect of the adaptation for the stiffness ellipsoid in the two-dimensional case. A large position error $\|\mathbf{e}(t)\|$ indicates contact of the manipulator or the grasped DLO with the environment. By applying the variable admittance parameters the system becomes more compliant in the directions of the tracking error, i.e. in constrained directions, whereas the basic compliance in unconstrained directions results in a good tracking performance. Mass and damping are also adapted to keep the system stable and well-damped.

\footnotetext{
${ }^{1}$ If $\|\mathbf{e}(t)\|=0$ or $\mathbf{e}(t)$ is in the direction of the $\mathrm{x}$-axis, then $R(\mathbf{e}(t))$ is the identity matrix.
}

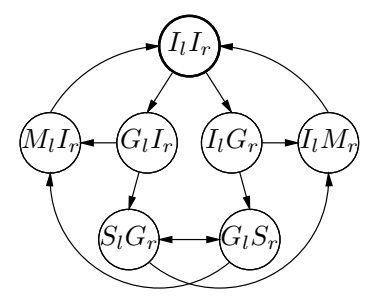

Fig. 2. State machine with possible state transitions for the DLO mounting task.

The potential large tracking errors of this system are reduced by an error-dependent time-warping $\tau(t)$ of the reference trajectory's time index according to

$$
\begin{aligned}
\tau(0) & =0 \\
\tau(t) & =\tau(t-1)+\max \left\{\Delta \tau_{\text {min }}, \Delta \tau-p_{\tau}\|\mathbf{e}(t)\|\right\},
\end{aligned}
$$

with $\Delta \tau>0$ the nominal index increment when there is no position error $(\Delta \tau=1$ if the nominal increment corresponds to the increment of the demonstrated trajectory), $\Delta \tau_{\min }$ the minimal increment and $p_{\tau} \geq 0$ is a proportional gain. Accordingly, the task is performed faster when the actual situation closely corresponds to the recording, whereas the execution speed is reduced under large deviations increasing task success rate.

\section{B. Automated Plan Extraction}

In an off-line step we analyze the recorded data in order to extract the plan for the given task. Therefore, we define a set with a finite number of discrete states $\mathcal{S}=\{G, S, M, I\}$ in which each state represents one of the above control primitives given by a desired trajectory and an appropriate control law.

Based on the state definition a time series of discrete states $\mathbf{S}_{l}=\left\{s_{l, t}: t \in\{0, \cdots, T\}, s_{l, t} \in \mathcal{S}\right\}$ for the states of the left manipulator and analogously $\mathbf{S}_{r}$ for the right manipulator is obtained. We define the combined state $s_{t}=\left(s_{l}, s_{r}\right) \in \mathcal{S}^{2}$ to compactly describe the system state in the bi-manual system. A state $s_{t}=\emptyset$ means the state for time $t$ is not defined yet. Admissible state transitions are defined according to expert knowledge of the task. In Fig. 2 the combined state machine for both manipulators is shown.

An algorithm based on heuristic rules is used to extract the state sequences $\mathbf{S}_{l}$ and $\mathbf{S}_{r}$ from the time series of grasping points $Y_{l}$ and $Y_{r}$, where the indexes $l$ and $r$ indicate the left or the right arm. It is executed once for each arm and exemplary given for the left manipulator in the flowchart diagram in Fig 3. During the execution the sequence $\mathbf{S}$ is used to determine the next control primitive.

\section{EXPERIMENTAL VALIDATION}

In order to validate the automatic state extraction and the variable admittance control scheme (in comparison to constant admittance) a tube mounting task using an autonomous robot is considered. It involves bi-manual manipulation of the deformable object, multiple contact phases with the deformable object and the rigid environment including pegin-the-hole type tasks, and multiple grasping primitives. 


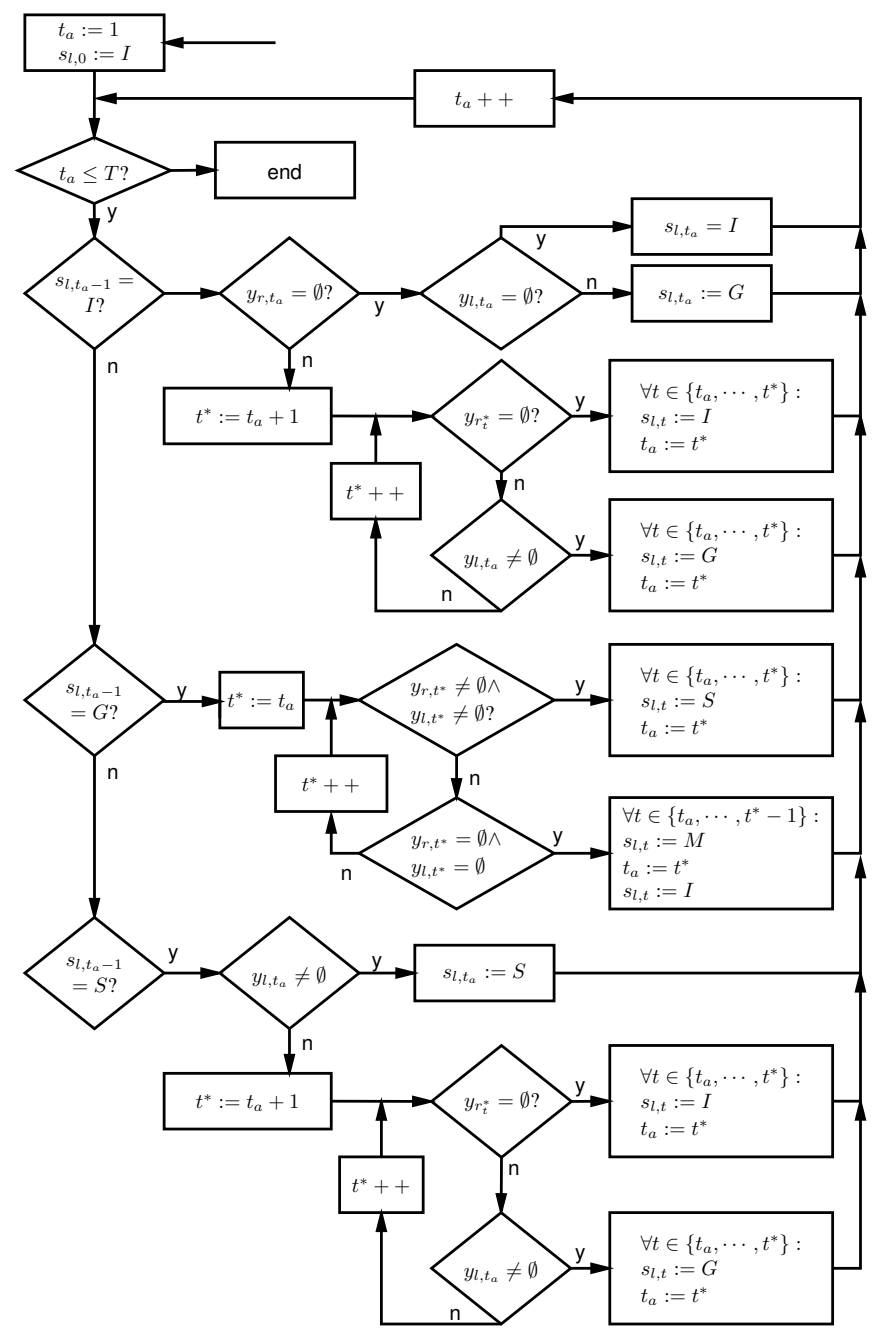

Fig. 3. Flowchart of the state extraction algorithm.

The testbed can be seen in Fig. 4. The goal of the manipulation task is to insert the tube through the two constraints and bring it to the final configuration. In this section we briefly describe the experimental setup before presenting our experimental procedure and obtained results.

\section{A. Setup}

The hardware setup used in the experiment consists of an autonomous robot which is also used as teleoperator, a human-system interface, and a central tracking system. These systems are described in the following. Furthermore, the used software architecture, the controller used in teleoperation mode, as well as the approach used for vision based grasping are briefly presented.

1) Teleoperator and Autonomous Robot: The robot is shown in Fig. 5(a). The main components are a camera head, two arms with grippers and a mobile platform. The camera head is equipped with a pair of wide-angle stereo cameras and a RGB-D camera (Mircosoft Kinect) providing depth information. The head itself is a pan tilt unit, but for the experiment we keep the head pose fixed. A pair of 7DoF anthropomorphic arms is mounted on the robot main chassis providing a human-like working space [6]. Attached

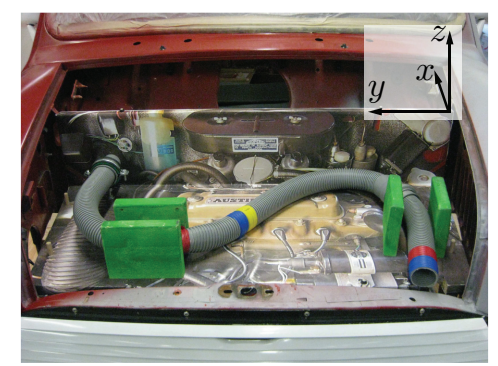

Fig. 4. The setup for the tube mounting task. The goal of the task is to bring the tube from a starting configuration to the depicted final configuration by grasping and manipulating the tube at the two-colored markers.

to each arm is a JR3 force-torque sensor and a Schunk PG70 two-finger gripper. The main chassis is based on a fourwheeled omni-directional mobile platform [7]. For relative localization of the robot with respect to the setup we use the LED-based Visualeyez ${ }^{\mathrm{TM}}$ VZ4000 motion measurement and tracking system by PhoeniX Technologies Inc.

2) Human-System Interface: For the teach-in we use a stationary human-system interface presented in [8] which is shown in Fig. 5(b). The images from the robot's camera pair are displayed via a head-mounted display. This grants the expert a natural view of the scene. The robot manipulators are coupled to two hyper-redundant haptic interfaces VISHARD10 [9]. These cover a human-like workspace and can display high impedances as are present in the examined task. Finger flection is captured by CyberGloves in order to control the robot grippers.

3) Software Architecture: Our real-time control framework [10][11], which is based on the KogmoRTDB real-time database [12] is used during demonstration and autonomous task execution. In addition to synchronized data management it allows to record and replay data streams.

4) Control in teleoperation mode: In teleoperation mode a bilateral control architecture couples the master and the slave. Hereby, we use position-based admittance controllers which are well suited to compensate the non-linear dynamics of an admittance-type haptic interface or robot [9]. On the master side a position-based admittance controller with force input is used and on the slave side a position-based admittance controller with position input. Using this architecture, forces from the master side and the slave side are both applied to one virtual admittance. Position control is then used to track the position of the virtual admittance on the master side. On the slave side, an additional impedance allows for some compliance when interacting with high impedance environments. The architecture runs at a sampling rate of $1 \mathrm{kHz}$. For more details on this control architecture see [13].

5) Vision Based Grasping: The grasping is implemented as look-and-move position control using the artificial markers on the DLO. The colored markers are detected with the RGB-D camera unit. As the markers are rotation-symmetric only the longitudinal axis $\mathbf{n}$ is known and the grasping pose is under-determined. In order to solve this problem, the grasping pose is chosen to be as close as possible to to the pose of the recorded reference. Given the rotation matrix from world coordinates to end-effector coordi- 


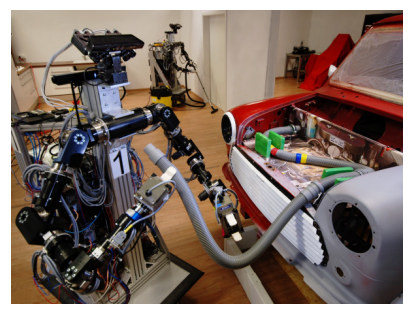

(a)

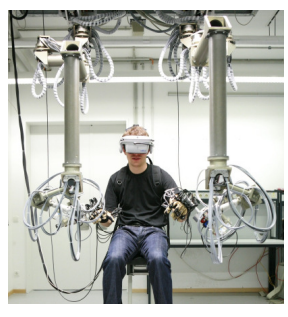

(b)
Fig. 5. The robot used in the experiment (a) and the two VISHaRD10 haptic interfaces [9] used for tele-operation (b).

nates ${ }^{0} \mathbf{R}_{E}^{\prime}=\left(\begin{array}{lll}\mathbf{n}^{\prime} & \mathbf{s}^{\prime} & \mathbf{a}^{\prime}\end{array}\right)$ of the reference grasp, where $\mathbf{n}^{\prime}$ is the reference longitudinal axes of the tube ${ }^{2}$, the desired transformation ${ }^{0} \mathbf{R}_{E}=\left(\begin{array}{lll}\mathbf{n} & \mathbf{s} & \mathbf{a}\end{array}\right)$ is obtained by

$$
\begin{aligned}
& \mathbf{s}=\frac{\mathbf{s}^{\prime}-\left(\mathbf{s}^{\prime} \cdot \mathbf{n}\right) \mathbf{n}}{\left\|\mathbf{s}^{\prime}-\left(\mathbf{s}^{\prime} \cdot \mathbf{n}\right) \mathbf{n}\right\|} \text { and } \\
& \mathbf{a}=\mathbf{n} \times \mathbf{s} .
\end{aligned}
$$

The marker position and the rotation defined by ${ }^{0} \mathbf{R}_{E}$ are used as the setpoint for the position control. This choice has the benefit of avoiding additional torsion compared to the demonstration.

\section{B. Procedure}

The DLO used for the experiment is a flexible tube made of silicone rubber with a waved surface. Plastic deformations are negligible. It has an outer radius of $R=20 \mathrm{~mm}$, a length of $1.25 \mathrm{~m}$, and a mass of $1.25 \mathrm{~kg}$. The two constraints used consist of two parallel walls of length $0.12 \mathrm{~m}$ and height $0.10 \mathrm{~m}$. The distance of two corresponding walls is $0.05 \mathrm{~m}$.

In a first step, a user demonstrates the task using teleoperation. During the demonstration, the robot's mobile base position is fixed to not introduce additional variance in the recorded data due to base motion. The complete robot state including manipulator positions and forces, gripper states and the poses of the robot and the testbed is stored during demonstration using the KogmoRTDB. The markers are subsequently applied to the DLO at the positions the user was grasping. The plan extraction is applied to the recorded data yielding the state sequence and the sequence of control primitives.

Next, the DLO insertion task is executed autonomously. The standard parameters $m, d$ and $k$ are set to $m=8 \mathrm{~kg}$, $d=600 \mathrm{Ns} / \mathrm{m}, k=400 \mathrm{~N} / \mathrm{m}$. The rotational admittance parameters are set to $\mathbf{M}_{o}=\operatorname{diag}\left(\begin{array}{lll}0.3 & 0.3 & 0.3) \\ \mathrm{Nms}^{2}, \mathbf{D}_{o}=\end{array}\right.$ $\operatorname{diag}(404040)$ Nms and $\mathbf{K}_{o}=\operatorname{diag}(404040) \mathrm{Nm}$. Three different conditions for the variable admittance parameter gains in (3) are evaluated (see Table I). The three conditions are labeled zero for fixed parameters, normal for moderate values, and high for gains five times the moderate ones. The parameters in (4) controlling the execution speed are set to $\Delta \tau=1.2, \Delta \tau_{\min }=0.2$ and $p_{\tau}=10 \mathrm{~m}^{-1}$. The robot platform is positioned once for all conditions in order to yield comparable results. The accuracy of positioning is

\footnotetext{
${ }^{2}$ Here we assume, the demonstrating user aligns the end-effector with the tube.
}

\begin{tabular}{cccc}
\hline condition & $p_{m}(\mathrm{~kg} / \mathrm{m})$ & $p_{d}\left(\mathrm{Ns} / \mathrm{m}^{2}\right)$ & $p_{k}\left(\mathrm{~N} / \mathrm{m}^{2}\right)$ \\
\hline zero & 0 & 0 & 0 \\
normal & 100 & 500 & 1000 \\
high & 500 & 2500 & 5000 \\
\hline
\end{tabular}

TABLE I

PARAMETER SETS USED FOR THE EXPERIMENTS.

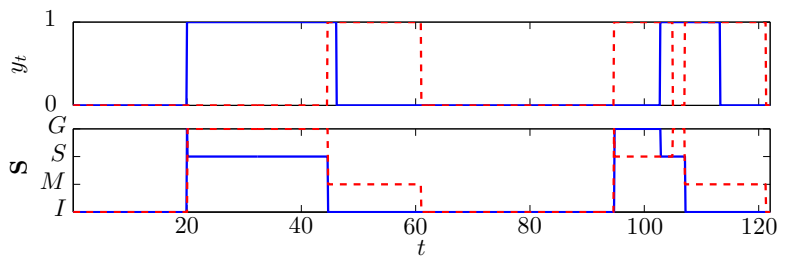

Fig. 6. Grasping sequence (top) from the demonstration and extracted states (bottom) for the left (solid) and the right (dashed) manipulator.

chosen to be $\pm 0.01 \mathrm{~m}$ for the position and $\pm 0.001 \mathrm{rad}$ for the orientation. Furthermore, the tracking system has a variance of $\pm 0.005 \mathrm{~m}$.

\section{Results}

The demonstrating user performs the task in about $121 \mathrm{~s}$. The extracted state sequence is $S=$ $\left(I_{l} I_{r}, G_{l} I_{r}, S_{l} G_{r}, I_{l} M_{r}, I_{l} I_{r}, I_{l} G_{r}, G_{l} S_{r}, S_{l} G_{r}, I_{l} M_{r}, I_{l} I_{r}\right)$. The grasping sequence from the demonstration and the extracted series of discrete states can be seen in Fig. 6 .

The autonomous task execution is performed twice with each parameter set. For each trial, Table II shows the execution time and the success of insertion into the two constraints. Note that the execution time is not directly comparable to the human demonstration, as waiting periods of several seconds occurred when searching in the recorded data.

\begin{tabular}{ccccc}
\hline condition & trial & time & constraint 1 & constraint 2 \\
\hline zero & 1 & $137 \mathrm{~s}$ & fail & fail \\
zero & 2 & $138 \mathrm{~s}$ & fail & fail \\
normal & 1 & $143 \mathrm{~s}$ & success & fail \\
normal & 2 & $140 \mathrm{~s}$ & success & success \\
high & 1 & $198 \mathrm{~s}$ & success & fail \\
high & 2 & $196 \mathrm{~s}$ & success & fail \\
\hline
\end{tabular}

TABLE II

QUALITATIVE RESULTS OF THE EXPERIMENTS.

\section{Discussion}

A clear advantage of the variable admittance controllers (conditions normal and high) is evident compared to the fixed admittance controller (condition zero) as latter is never successful at inserting the tube into a constraint. This suggests that the fixed admittance controller is not able to overcome the uncertainties introduced by inexact positioning of the platform, the inaccurate vision-based grasping, and other noisy data.

However, a task-appropriate choice of the variable admittance parameters is crucial as observable from the decreased performance for the condition high compared to the condition normal. With this parametrization, the actual trajectory drifts 


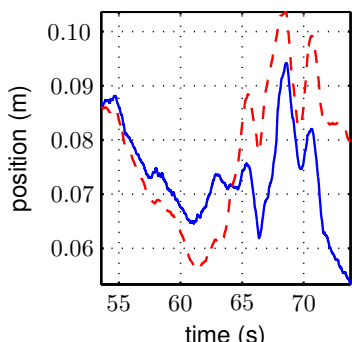

(a)

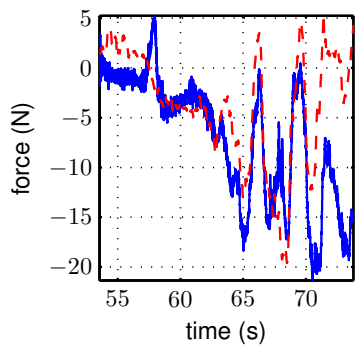

(c)

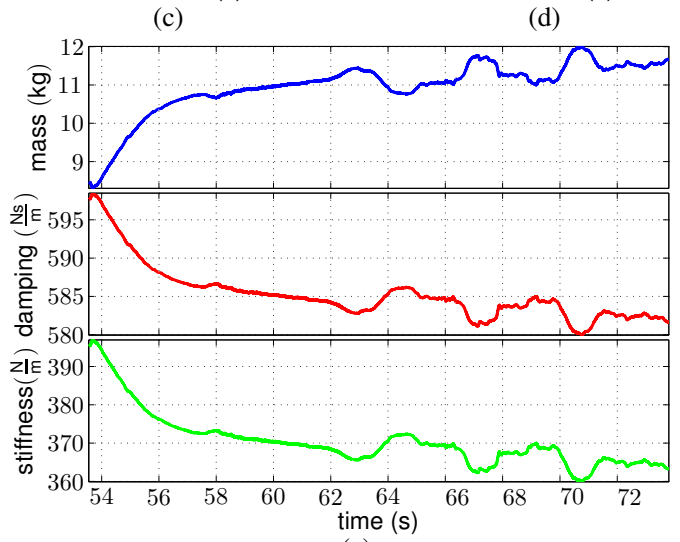

(e)

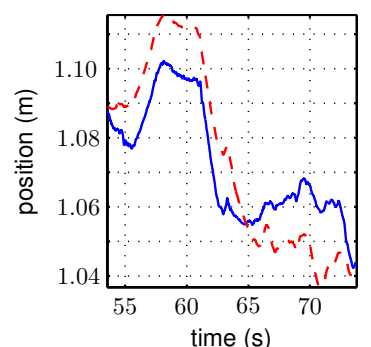

(b)

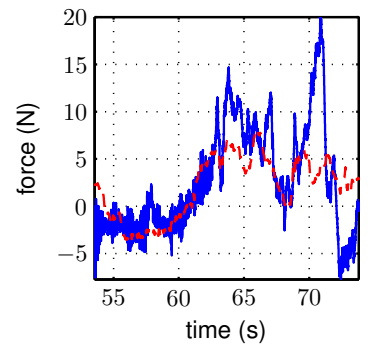

(d) 7(a) to 7(d) show the $y$ - and the $z$-coordinates of the according pose and wrench trajectories of the haptic control primitive $\mathbf{P}_{1}$ (dashed lines) and the actual trajectories performed by the robot (solid lines). 7(e) shows the evolution of the admittance parameters.

away from the desired trajectory, making a successful task execution impossible.

In Fig. 7 trajectories and admittance parameters for the insertion of the DLO into the first constraint are shown for the first trial of the condition normal. The similar shape of the desired and the actual trajectories indicate that the desired position and force is compliantly tracked. Between $65 \mathrm{~s}$ and $73 \mathrm{~s}$ the tube is pushing against the obstacle in negative $z$-direction (i.e. down) which is mainly visible in the positive force in $z$-direction. It can be observed that movement in negative $z$-direction (i.e. slipping into the constraint) occurs at a different time for the recorded case and autonomous insertion which is probably due to positioning errors of the robot. Repeated successfull insertion using our variable admittance controller indicates that the controller is well suited to handle DLO insertion tasks with such uncertainties. Fig. 7(e) shows an example of the evolution of the admittance parameters starting at the standard values for $m, d$ and $k$.

\section{CONCLusions}

In this paper we present a systematic approach for transferring a complex deformable object manipulation task from a human demonstration to an autonomous robot. By analyzing the demonstration sequence a task plan is obtained consisting of the sequence of control primitives. The control primitives consist of a reference trajectory (or setpoint) and an appropriate feedback control. In order to cope with positioning uncertainties in contact phases, we propose an error-dependent admittance control scheme, which reduces the compliance in the direction of the error occurring from environment constraints. As a result, high tracking accuracy is achieved in free-space motion while providing limited internal forces and stability in contact situations. The approach is validated in a bi-manual mounting task for deformable objects. For the future, a systematic robustness analysis with respect to positioning errors and a stability proof is planned.

\section{REFERENCES}

[1] H. Wakamatsu and S. Hirai, "Static modeling of linear object deformation based on differential geometry," I. J. Robotic Res., vol. 23, no. 3, pp. 293-311, 2004.

[2] H. Wakamatsu, T. Yamasaki, S. Hirai, A. Tsumaya, and E. Arai, "Dynamic modeling of linear object deformation considering contact with obstacles," in Control, Automation, Robotics and Vision, 2006. ICARCV '06. 9th International Conference on, dec. 2006, pp. 1 -6.

[3] M. Saha and P. Isto, "Manipulation Planning for Deformable Linear Objects," IEEE Transactions on Robotics, vol. 23, no. 6, pp. 11411150, Dec. 2007.

[4] K. Hirana, T. Suzuki, S. Okuma, K. Itabashi, and F. Fujiwara, "Realization of skill controllers for manipulation of deformable objects based on hybrid automata," in Robotics and Automation, 2001. Proceedings 2001 ICRA. IEEE International Conference on, vol. 3. IEEE, 2001, pp. 2674-2679. [Online]. Available: http://ieeexplore.ieee.org/xpls/abs_all.jsp?arnumber=933026

[5] J. Acker and D. Henrich, "Manipulation of deformable linear objects: From geometric model towards program generation," in Robotics and Automation, 2005. ICRA 2005. Proceedings of the 2005 IEEE International Conference on, april 2005, pp. 1541 - 1547.

[6] B. Stanczyk and M. Buss, "Development of a Telerobotic System for Exploration of Hazardous Environments," in Proc. IEEE/RSJ IROS, 2004, pp. 2532-2537.

[7] U. D. Hanebeck, N. Saldic, and G. Schmidt, "A modular wheel system for mobile robot applications," in Proceedings of the IEEE/RSJ International Conference on Intelligent Robots and Systems (IROS), 1999 , pp. 17-23.

[8] M. Buss, A. Peer, T. Schauss, N. Stefanov, U. Unterhinninghofen, S. Behrendt, J. Leupold, M. Durkovic, and M. Sarkis, "Development of a multi-modal multi-user telepresence and teleaction system," Int. J. Rob. Res., vol. 29, pp. 1298-1316, September 2010.

[9] M. Ueberle, N. Mock, and M. Buss, "Vishard10, a novel hyperredundant haptic interface," in Haptic Interfaces for Virtual Environment and Teleoperator Systems, 2004. HAPTICS '04. Proceedings. 12th International Symposium on, march 2004, pp. $58-65$.

[10] D. Althoff, O. Kourakos, M. Lawitzky, A. Mörtl, M. Rambow, F. Rohrmüller, D. Brščić, S. Hirche, and M. Buss, "An architecture for real-time control in multi-robot systems," in 3rd International Workshop on Human-Centered Robotic Systems, 2009.

[11] M. Rambow, F. Rohrmüller, O. Kourakos, D. Brščić, D. Wollherr, S. Hirche, and M. Buss, "A framework for information distribution, task execution and decision making in multi-robot systems," IEICE Transactions on Information and Systems, vol. E93-D, no. 6, pp. 13521360, 2010.

[12] M. Goebl and G. Färber, "A real-time-capable hard- and software architecture for joint image and knowledge processing in cognitive automobiles," in Proceedings of the 2007 IEEE Intelligent Vehicles Symposium, June 2007, pp. 734-740.

[13] A. Peer and M. Buss, "Robust stability analysis of a bilateral teleoperation system using the parameter space approach," in IEEE/RSJ International Conference on Intelligent Robots and Systems, 2008, pp. $2350-2356$. 